\title{
ATITUDE PEDAGÓGICA DECOLONIAL
}

\author{
Nelson Wanahowa Karajá1
}

\section{RESUMO}

Otrabalho, na minha prática pedagógica do estágio, é um trabalho constituído de temas contextuais inter e intraculturais que foram desenvolvidos através da pesquisa nas aldeias. O trabalho desenvolvido no estágio tem como finalidade resgatar e fortalecer a nossa cultura entre os jovens na escola e na comunidade. São temas contextuais de fortalecimento culturais. O estágio pedagógico contém vários temas contextuais que atendem demandas da minha comunidade. São experiências minhas de ensino e aprendizagem na escola e na comunidade. Uma experiência de várias descobertas que mudou o meu olhar em relação à minha cultura. $\mathrm{O}$ meu estágio tem como princípios pedagógicos a interculturalidade e a transdiciplinaridade.

PALAVRAS-CHAVE: Estágios pedagógicos. Experiências. Resgate e fortalecimento cultural.

\section{TURYBÈNA BUTÈ}

Waõmysỹdỹỹna rèòrarunyre-u hãwãhãwã-my rèare tyyrti ariwahinykrekoki, matuari, senado bdèèry mahãdù mynamyna rariare ryyraxi-my, waõmysỹdỹỹna iny bdèdỹỹnana-my tyyrti idi ãriwahinykre-my. Inatão bèòra-di iruyrè-my aõmysỹdỹỹna remyre tyyrti idi ariwahinykre-my iny bdèdỹỹnana-my. Kaa waõmysỹdỹỹna rewinyre iny bdèdỹỹnana idi ãritnynynykre-my, ijoi tùù arinohõti ijranykre-my, timybo juhu-u iny hỹỹna reny rỹiramyhỹ-my dỹỹraxi rbi rèèry rahù-di tyyrti rèwahinyre. Tule tori bdèdỹỹnana-my tyyrti rèwahinyre, timybo rybè rèlèmyhỹre-my tyyrti rèwahinyre. Waõmysỹdỹỹna rèwinyre tyyrti dỹỹ-du tyhy-my ãrelekremy,waijoi ariwiòhènanykre-my. Myy waijyy rèwinyre dỹỹraxi rbi. Iny bdèdỹỹnana ãrierykre-my, irbi tahè ùladù ãrièrynanykre-my tyyrtina wo-ki.

1 Licenciado no Curso de Educação Intercultural pela Universidade Federal de Goiás. Professor da Educação Básica. Escola Estadual Indígena Tapirapé. Pesquisador da Ação "Saberes Indígenas na Escola", Rede UFG/UFT/ UFMA. Aldeia Mayteritawa, MT, Brasil. E-mail: wananelson@gmail.com. 


\section{ABSTRACT}

The work in my pedagogical practice of the stage, is a work composed of contextual themes Inter and intracultural that was developed through the research in the villages. The work carried out at the internship is aimed at rescuing and strengthening our culture among the youth in school and in the community, are contextual themes of cultural strengthening. The pedagogic stage contains several contextual themes that meet the demand of my community, are my experiences of teaching and learning in school and in the community. An experience of several discoveries that changed my look regarding my culture. My internship has pedagogical principles of interculturality and transdisciplinarity.

KEYWORDS: Pedagogical internships. Experiences. Rescue and cultural strengthening.

\section{Apresentação}

Neste artigo, apresento como aconteceu meu estágio pedagógico no curso de Educação Intercultural da Universidade Federal de Goiás. Foram dois anos e meio de estágio. Durante esse período, experimentei trabalhar com temas contextuais intraculturais, interculturais e, até mesmo, transculturais, fato que é possível quando problematizamos o tema e ele se estica em dimensão política e epistêmica. Sempre fiz um plano de estudo que se constituía de leituras e pesquisa, uma orientação dada pela nossa orientadora Maria do Socorro Pimentel da Silva. Aprendi nos meus estágios a ser um professor preocupado com a valorização do nosso saber, mas também a articular saberes, pois a escola de indígena precisa dos dois saberes, do indígena e do não indígena. Por isso, meu trabalho está baseado em autores Karajá e em Pimentel da Silva (2017).

\section{Meu Estágio I}

No primeiro estágio, escolhi um tema intracultural sobre a classificação tradicional de animais. Escolhi este tema porque é um tema que é extremamente importante para meu povo. As crianças precisam aprender como antigamente os Iny/ Karajá classificavam os animais. Antes de tudo, fiz a minha pesquisa na comunidade Karajá, 
aldeia Itxala e aldeia Hawalora. Pesquisei vários anciãos e anciãs e também os professores da Escola Estadual Indígena Hawalora. A minha linha de pesquisa foi em relação à classificação de animais, de como antigamente os Iny classificavam os animais. Descobri na pesquisa que os Iny tinham uma classificação diferente em relação à classificação científica. Nosso conhecimento se organiza de modo bem diferente das ciências ocidentais. Antes do curso da Educação Intercultural, nosso conhecimento era apagado na escola.

No meu primeiro estágio, fiz uma aula teórica com os alunos da EJA. Aprendi muitas coisas com eles e foi a minha primeira experiência na sala de aula. Nesse trabalho, descobri que a pesquisa não foi apenas para eu fazer a minha prática pedagógica, mas sim foi uma prática de retomada de saberes, como bem afirma Pimentel da Silva (2016). Só pude perceber isso no andamento do estágio. Fiz juntamente com os alunos a discussão sobre várias espécies de animais.

\section{A palavra "animais", na língua Karaja é IRÒDÙ}

Nessa pesquisa descobri que nós, Iny, temos apenas três grandes grupos de animais: IRÖDÙ (animais), NAWII (aves), UTURA(peixe). Esses são os três grandes grupos. Além disso, existem outras espécies de seres vivos, como abelhas, formigas, camaleão, tartaruga, jacaré e outras espécies de animais que são conhecidas apenas pelos nomes, não fazem parte desses grandes grupos. Assim, fiz o meu primeiro estágio com vários questionamentos e foi uma discussão riquíssima de reflexões. Percebi como nós, professores, estamos adotando uma educação decolonizadora dentro das nossas escolas indígenas e, assim, ensinando as nossas crianças a pensarem sobre a nossa realidade primeiro. Precisamos discutir mais sobre uma educação escolar indígena, incluir mais a realidade indígena na escola, para que ela seja valorizada pelos jovens.

\section{Estágio II}

No estágio II, trabalhei com o tema contextual ÃXI (uma pescaria coletiva do povo Iny/ Karajá). Primeiramente, fiz uma pesquisa, depois planejei a minha prática pedagógica na sala de aula. Fiz a minha pesquisa nas aldeias Hawalora e na aldeia Majtyri sobre 
o ÃXI, como antigamente os Iny faziam a pescaria com timbó e, nessa pesquisa, descobri várias coisas sobre essa pescaria e como os Iny faziam essa prática e como a praticam hoje.

Busquei as informações adotando as seguintes linhas de pesquisa. Usei na pesquisa com os entrevistados as seguintes perguntas:

- Origem do ãxi, como os Iny descobriram o cipó?

- Como era essa prática?

- Quem pode fazer, homem ou mulher?

- Quais as divisões de trabalho?

- Qual o tempo de bater timbó?

Essa linha de estudo, usei para me orientar durante a minha pesquisa. Depois da minha pesquisa, levei os vários dados coletados para a sala de aula. Primeiro, juntamente com os alunos, fizemos uma aula teórica de acordo com o plano de aula. Fizemos um levantamento de tudo que é necessário para fazer o ÃXI. Mas, percebi que a aula teórica não foi uma aula produtiva, então tive um papel de proporcionar aos alunos uma amostra de como essa pescaria é praticada. Nesse estágio, tive a participação de todas as comunidades e alunos das aldeias. Fiz um planejamento de três dias.

No primeiro dia, fizemos as flechas.

No segundo dia, com todos os alunos (meninos) e membros da comunidade fomos pegar o timbó na mata.

No terceiro dia, fizemos a pescaria no lago. 
Foto 1 - Orientação para coletar timbó no mato. Timbó tem a função de facilitar a captura de peixes para alimentar a comunidade.

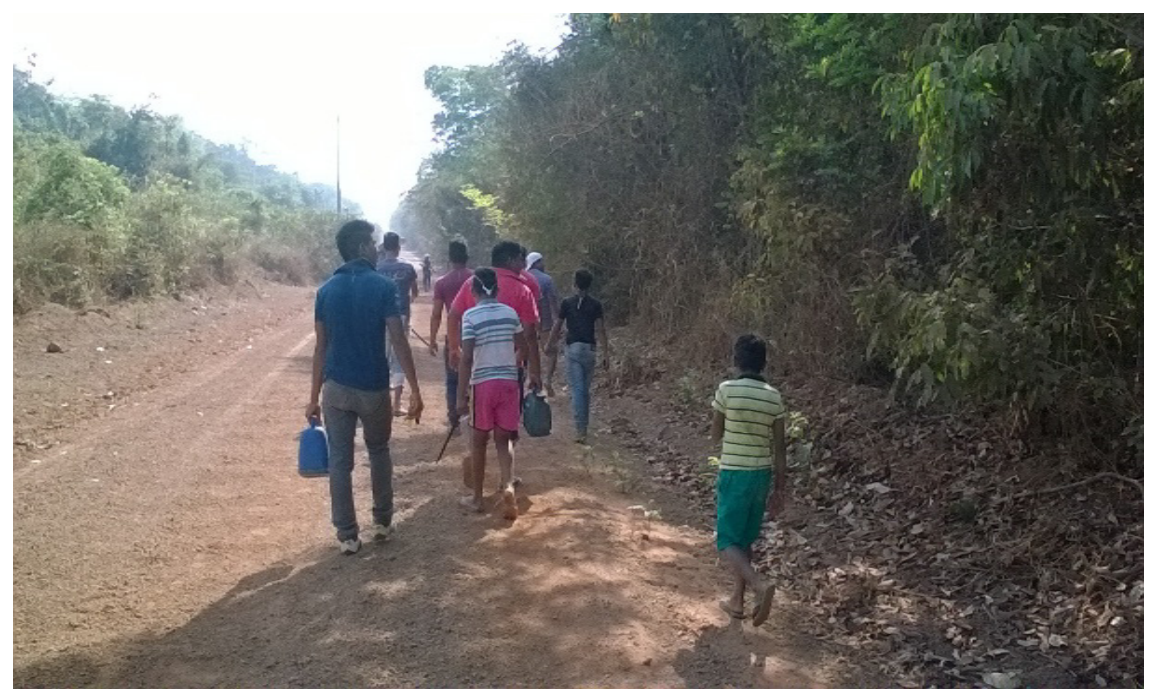

Fonte: Nelson Wanahowa Karajá.

Foto 2 - Os meninos são orientados para tirar o cipó com cuidado. Depois de tirar os cipós, cortam os tamanhos iguais e carregam para o local escolhido, ou seja, um lago tamanho pequeno.

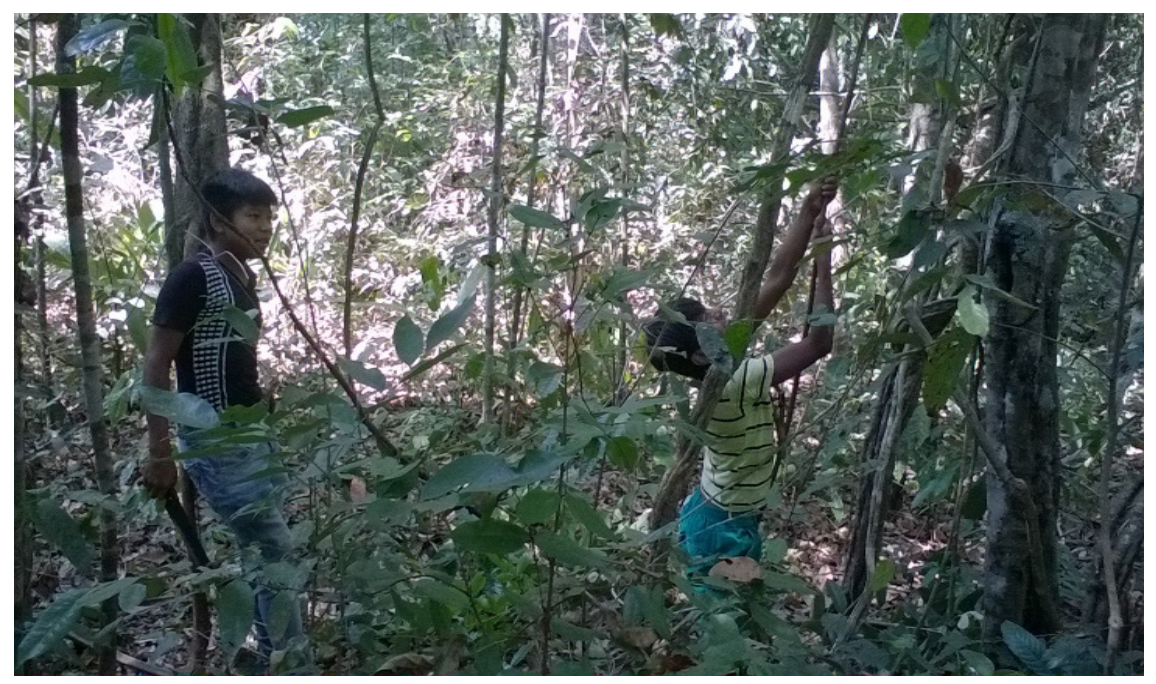

Fonte: Nelson Wanahowa Karajá. 
Então, nesse estágio, fiz uma aula teórica e uma aula prática. No estágio II, tive a percepção de que uma aula teórica é importante, mas um conhecimento Iny não pode ficar apenas na teoria. Ao se trabalhar com tema contextual intracultural, como ãxi, é um tema que pode ser tanto na teoria quanto na prática. Temas assim envolvem não só os alunos, mas também a comunidade.

Foto 3 - Pescando com timbó.

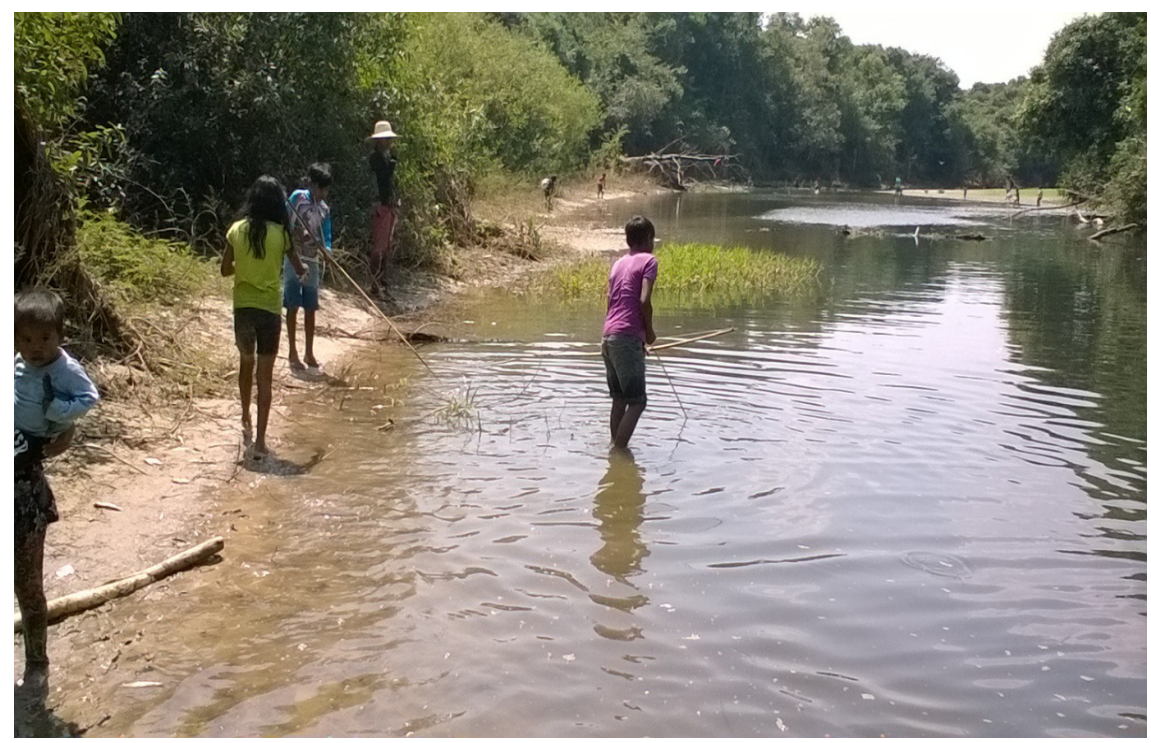

Fonte: Nelson Wanahowa Karajá.

O estágio II me proporcionou pesquisa e, através dela, tive várias descobertas interessantes em relação à pescaria com timbó. Isso foi essencial durante a minha prática pedagógica no estágio.

\section{Estágios III e IV}

No estágio III, trabalhei com tema contextual: derivação das palavras em língua portuguesa, esse tema contextual foi um dos maiores desafios em relação a tudo. Antes das minhas práticas pedagógicas na sala de aula, fiz a minha pesquisa na internet, nos livros de língua portuguesa e com os outros professores mais experientes. Esse tema contextual, "Derivação das palavras", teve 
como objetivo que nós, professores, abordássemos o conhecimento não indígena, como as palavras são formadas.

Fiz a minha pesquisa colhendo várias informações. Depois, fiz o meu plano de aula para trabalhar com elas na sala de aula. Nesse estágio, tive como meu objetivo mostrar para os alunos como as palavras são formadas. Usei como exemplo o que aprendi durante a minha pesquisa. Que existem dois processos básicos pelos quais se formam as palavras; derivação e composição. No estágio III, abordei apenas as derivações prefixal e sufixal, ou prefixação e sufixação. Exemplos que utilizei durante a minha prática pedagógica do estágio.

\section{Derivação prefixal ou prefixação:}

- $\quad$ Feliz $=$ infeliz

- $\quad$ Terra $=$ enterrar

- Ler $=$ reler

\section{Derivação sufixal ou sufixação:}

- Terra $=$ terreiro

- $\quad$ Feliz = felizmente

- $\quad$ Pedra $=$ pedreiro

\section{Derivação sufixal e prefixal:}

- Infelizmente

Esses são alguns exemplos que utilizei durante o meu estágio. Mas, percebi que só o estágio III não seria suficiente para trabalhar com um tema contextual dessa proporção, ou seja, 5 aulas, com o tema bem contextualizado. Percebi que podia continuar o trabalho no estágio IV. Então, continuei o meu estágio sobre derivação das palavras, para um melhor aperfeiçoamento do meu estágio pedagógico. Isso não foi bom só para os alunos, mas sim para mim também, pois na minha formação tive várias dificuldades. Primeiro, por conta de que a língua portuguesa não é a nossa primeira língua. Segundo, vi que os alunos tiveram muitas dificuldades, mas, apesar disso, vi também que eles gostaram desse tema contextual. 
Foto 4 - Atividades escritas da aula.
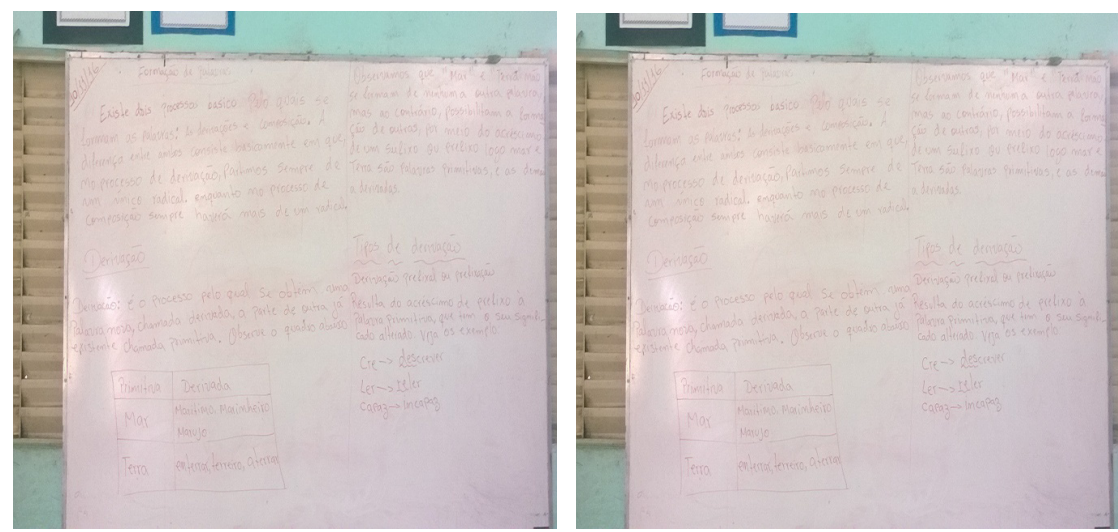

Fonte: Nelson Wanahowa Karajá.

Assim foram os meus estágios III e IV, na sala de aula, com os alunos em relação à língua portuguesa. Um tema rico em discussão, tanto na língua portuguesa quanto na língua Iny Karajá. Por isso, esse tema foi muito produtivo na sala de aula.

\section{Estágio V}

No estágio V, trabalhei com o tema contextual "Mensageiros da natureza", um tema intracultural, que escolhi porque percebi durante uma visita na aldeia Itxala, onde uma senhora Iny estava contando que ela teve um presságio através de um pássaro que lhe contou que aconteceria um evento importante na aldeia. Isso motivou muito a escolha do tema contextual. Percebi ali que nós, jovens, estávamos esquecendo das mensagens vindas da natureza.

Então, comecei a minha pesquisa como sempre vinha fazendo na minha prática pedagógica do estágio. Primeiro, fiz a minha pesquisa nas aldeias Majtyri, Hawalòra e Itxala. Nessas aldeias, colhi várias informações importantes em relação às mensagens da natureza.

A pesquisa nas aldeias é fundamental para elaborar o plano de aula e também para promover várias outras pesquisas. Pesquisa é uma descoberta, e um aprendizado para quem pesquisa e também é um momento de relembrar o conhecimento adormecido dos anciãos. $\mathrm{E}$, nessa pesquisa, descobri que as mensagens que a natureza traz 
vêm de várias formas. Através de várias espécies de ser vivo ou em forma de espíritos.

De acordo com o plano de aula, primeiro fizemos juntamente com os alunos, as listagens de mensageiros: animais, aves, plantas, espírito e outros seres.

Lista do nome de mensageiros da natureza nas falas feminina e masculina, respectivamente:

- Wekò = weò

- $\quad$ Ijybikò = ijybiò

- $\quad$ Xika $=$ xika

- $\quad$ Kybdò = ybdò

- $\quad$ Itxòròsa $=$ ijòròsa

- $\quad$ Kotxixa = otxixa

- $\quad$ Jyijyi = jyijyi

- Hawkynimyraha= Hawyynimyraha

- Halokòè = Halòè

- Itxòrò = ijòrò

- $\quad$ Ijybikò= ijybiò

- Kukasa

- Krauramysa= yrauramysa

- Halokoeni = haloeni

- Dorè

- $\quad$ Isidèrikò = isidèriò

- Raradòkò= raradòò

- Biri

Essas são as informações que colhi na pesquisa e que levei para sala de aula. Isso me ajudou muito durante a minha prática pedagógica. $\mathrm{O}$ estágio nos proporciona não apenas a nossa formação de professores, mas também, a valorização do nosso conhecimento. Pela minha percepção, o estágio não é apenas na sala de aula, ele é também praticado fora da sala de aula, na comunidade.

Assim, foi a minha prática pedagógica no estágio que fiz, todos na Escola Estadual Indígena Tapirapé com os alunos do ensino fundamental e EJA, alunos Karajá que vivem nessa aldeia. Durante meus estágios, percebi como é importante a educação intercultural crítica. Como é importante para a sobrevivência dos nossos conhecimentos, o trabalho com temas contextuais. Hoje me sinto professor. Sei pesquisar. A pesquisa é algo importante no 
aperfeiçoamento diário do professor. Sempre serei um professor transdisciplinar. Esta proposta faz o professor se comprometer com a retomada dos saberes milenares, mas também com a articulação de saberes em relação respeitosa.

\section{Referências bibliográficas}

KARAJÁ, Leandro Lariwana. Caderno de Estágio. Goiânia: UFG/Núcleo Takinahaky de Formação Superior de Professores Indígenas/Curso de Educação Intercultural, 2011.

KARAJÁ, Kuhanama. Caderno de Estágio. Goiânia: UFG/Núcleo Takinahaky de Formação Superior de Professores Indígenas/Curso de Educação Intercultural, 2011.

KARAJÁ, Txiarawa. Caderno de Estágio. Goiânia: UFG/Núcleo Takinahaky de Formação Superior de Professores Indígenas/Curso de Educação Intercultural, 2011.

PIMENTEL DA SILVA, Maria do Socorro. Pedagogia da retomada: descolonização de saberes. Revista Articulando e Construindo Saberes. Goiânia, v. 2, n. 1, p. 204-2015, 2017.

Recebido para publicação em abril de 2018.

Aceito para publicação em junho de 2018. 